\title{
Facile, Electrochemical Chlorination of Graphene from an Aqueous NaCl Solution
}

\author{
Wan Li, Yunqi Li, and Ke Xu ${ }^{*}$ \\ Department of Chemistry, University of California, Berkeley, California 94720, USA
}

\section{Supporting Information}

\section{Materials and Methods}

Preparation of monolayer graphene. Monolayer graphene grown on copper foils (ACS Material or Graphene Supermarket) was cut into $\sim 2 \times 10 \mathrm{~mm}$ strips, and wet-transferred onto silicon chips coated with a $300 \mathrm{~nm}$ thermal oxide layer $\left(\mathrm{SiO}_{2} / \mathrm{Si}\right)$ or \#1.5 glass coverslips, following the standard PMMA-based wet transfer method with a modified RCA cleaning process. ${ }^{1}$ After the removal of PMMA using acetone and isopropanol, the graphene strips were contacted at both ends using conductive silver paint.

Electrochemical chlorination of monolayer graphene. Best chlorination results were achieved in $1.5 \mathrm{M} \mathrm{NaCl}$ dissolved in a $200 \mathrm{mM} \mathrm{pH}=3$ potassium phosphate buffer. For examination of the $\mathrm{pH}$ dependence of chlorination efficiency, $1.5 \mathrm{M} \mathrm{NaCl}$ was dissolved in $18 \mathrm{M} \Omega$ Milli-Q water $(\mathrm{pH} \sim 6$ ) or a $200 \mathrm{mM} \mathrm{pH}=10$ sodium carbonate buffer. A small $(\sim 50-80 \mu \mathrm{L})$ drop of the $\mathrm{NaCl}$ solution was carefully placed at the center of the graphene strip without contacting the silver paint on either ends. $\mathrm{An} \mathrm{Ag} / \mathrm{AgCl}$ counter/reference electrode $(3 \mathrm{M} \mathrm{NaCl})$ was then inserted into the solution drop from the top to complete the electrochemical cell. A Keithley 2401 SourceMeter was programmed to apply voltages to graphene versus the $\mathrm{Ag} / \mathrm{AgCl}$ electrode and record the resultant electrochemical current. To avoid the potential electrochemical oxidation of graphene due to a long-lasting oxidative reaction voltage, chlorination was carried out in two cycles, with each cycle starting with a constant oxidative voltage in the range of 1.4-1.8 $\mathrm{V}$ for $2 \mathrm{~min}$, and then followed by a recovery period of $1 \mathrm{~min}$ at a reductive voltage at $0 \mathrm{~V}$ vs $\mathrm{Ag} / \mathrm{AgCl}$. The sample was then rinsed with Milli-Q water and dried in the air. For reaction in the mixed $\mathrm{NaCl}-\mathrm{NaN}_{3}$ solution, $1.5 \mathrm{M} \mathrm{NaCl}$ and $200 \mathrm{mM} \mathrm{NaN}_{3}$ were added into a $\mathrm{pH}=7$ phosphate buffer, and a constant oxidative voltage of $1.6 \mathrm{~V}$ (vs. $\mathrm{Ag} / \mathrm{AgCl}$ ) was similarly applied to the graphene electrode in two 2-min cycles followed by 1-min recovery periods at $0 \mathrm{~V}$. For sequential electrochemical chlorinationazidation, the graphene sample was first chlorinated at $1.6 \mathrm{~V}$ (vs. $\mathrm{Ag} / \mathrm{AgCl}$ ) in $1.5 \mathrm{M} \mathrm{NaCl}$ in a $\mathrm{pH}=3$ phosphate buffer as described above, rinsed with Milli-Q water, and then azidated at $1.3 \mathrm{~V}$ (vs. $\mathrm{Ag} / \mathrm{AgCl}$ ) in $200 \mathrm{mM} \mathrm{NaN}_{3}$ in a $\mathrm{pH}=7$ phosphate buffer for $5 \mathrm{~min}$.

Electrochemical bromination and iodination of monolayer graphene. The electrochemical cells were constructed similarly as above, except that $1 \mathrm{M} \mathrm{NaBr}$ and $1 \mathrm{M} \mathrm{KI}$ in $200 \mathrm{mM} \mathrm{pH}=3$ potassium phosphate buffers were used for bromination and iodination, respectively, and the counter/reference electrode was a Pt wire. A constant voltage of $1.6 \mathrm{~V}$ and $1.4 \mathrm{~V}$ vs. Pt was applied to graphene for $4 \mathrm{~min}$ for bromination and iodination, respectively. 
XPS and Raman characterizations. XPS spectra were obtained for samples on $\mathrm{SiO}_{2} / \mathrm{Si}$ substrates using a Perkin Elmer PHI 5600 XPS that operated at $\sim 10^{-9}$ torr with a monochromatic Al Ka $(1486.8 \mathrm{eV})$ X-ray source. The above samples were characterized by both survey-mode (0-1100 eV binding energy range) and high-resolution (15 or $20 \mathrm{eV}$ binding energy window around the peaks of interest) scans. The control $\mathrm{NaCl}$ solid sample was prepared by letting dry in the air a $\sim 150 \mu \mathrm{L}$ drop of $200 \mathrm{mM} \mathrm{NaCl}$ aqueous solution on a clean $\mathrm{SiO}_{2} / \mathrm{Si}$ substrate. For quantification, ${ }^{2}$ the Shirley's algorithm was applied to determine the background when calculating the XPS peak areas. $\mathrm{Cl}: \mathrm{C}, \mathrm{Br}: \mathrm{C}$, and $\mathrm{I}: \mathrm{C}$ atomic ratios were calculated from the peak areas from high-resolution XPS measurements using the factory-calibrated relative sensitivity factors. O:C atomic ratios were calculated from survey-mode XPS measurements using the factory-calibrated relative sensitivity factors after subtracting the oxygen component from the $\mathrm{SiO}_{2}$ substrate, which was calibrated to have a fixed O:Si atomic ratio of 2.02 \pm 0.01 . Raman spectra were recorded with a Renishaw InVia micro-Raman system using a $488 \mathrm{~nm}$ laser and a 2400 lines/mm grating.

Electrical and electrochemical characterizations. Graphene electrical properties were characterized through the contacts at the two ends of the graphene strip. A Keithley 2401 SourceMeter repeatedly scanned source-drain voltages through graphene in the range of -20 to $+20 \mathrm{mV}$, and a Keithley 2400 SourceMeter applied electrochemical gating voltages across the $\mathrm{Ag} / \mathrm{AgCl}$ electrode and graphene (electrolyte was a $200 \mathrm{mM} \mathrm{pH}=3$ phosphate buffer). Linear $I-V$ curves were obtained, from the slopes of which conductance was calculated. Cyclic voltammetry was carried out under a three-electrode configuration, using graphene as the working electrode, a Pt wire as the counter electrode, and an $\mathrm{Ag} / \mathrm{AgCl}$ electrode as the reference electrode. The Keithley 2401 SourceMeter was connected in a 4-wire configuration and programmed to sweep the voltage between the working and reference electrodes and record the resultant electrochemical current between the working and counter electrodes.

Interference reflection microscopy (IRM). For IRM, graphene samples were deposited on \#1.5 glass coverslips. IRM was performed as described previously ${ }^{3}$ on an Olympus IX73 inverted epifluorescence microscope using a UplanF1 100× oil-immersion objective lens (numerical aperture $\sim 0.9$ with iris diaphragm) and a white light source (Olympus U-HGLGPS). The excitation filter was D532/10x (Chroma), the dichroic mirror position was mounted with a 50/50 beam splitter (Chroma 21000), and the emission filter position was left empty. Wide-field images were recorded using an Andor Zyla 4.2 sCMOS camera. For in situ monitoring of the electrochemical oxidation of graphene, the IRM signal was converted into the local degree of oxidation, as discussed previously. ${ }^{4,5}$ Briefly, the IRM signal depends strongly on the imaginary part of the complex index of refraction of the sample, which is related to the conductivity of the material. The big difference in the refractive indices of graphene and graphene oxide at $n_{\mathrm{Gr}}=2.65+1.27 i$ and $n_{\mathrm{GO}}=1.75+0.17 i$ thus leads to substantial IRM contrast. When expressed as $C=$ $I / I_{0}, I$ and $I_{0}$ being the absolute IRM signals at the sample and at the bare glass substrate, respectively, $C_{\mathrm{Gr}}=0.73$ for the former and $C_{\mathrm{GO}}=0.97$ for the latter. The local oxidation degree at each pixel, with local IRM contrast measured as $C_{\text {pixel }}=I_{\text {pixel }} / I_{0}$, is thus estimated as: $:^{4,5}\left(C_{\text {pixel }}-C_{\mathrm{Gr}}\right) /\left(C_{\mathrm{GO}}-C_{\mathrm{Gr}}\right)$. 


\section{Supplementary Figures}
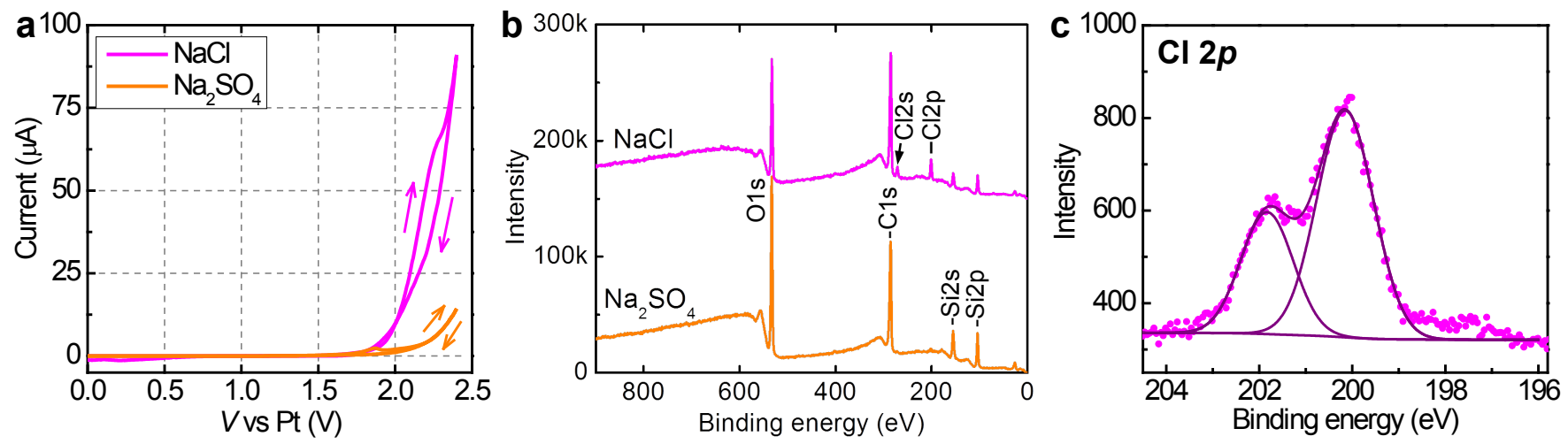

Figure S1. Electrochemical chlorination of graphene using a Pt wire as the counter/reference electrode. (a) Cyclic voltammograms measured vs. the $\mathrm{Pt}$ wire for monolayer graphene in $200 \mathrm{mM} \mathrm{pH}=3$ phosphate buffers with the addition of $1.5 \mathrm{M} \mathrm{NaCl}$ (magenta curve) vs. with the addition of $0.5 \mathrm{M} \mathrm{Na}_{2} \mathrm{SO}_{4}$ (orange curve). Scanning rate: 19 $\mathrm{mV} / \mathrm{s}$. (b) Survey-mode XPS spectra of two monolayer graphene devices on $\mathrm{SiO}_{2} / \mathrm{Si}$ substrates after electrochemical reactions at $2.1 \mathrm{~V}$ (vs. Pt) for $4 \mathrm{~min}$ in the above two solutions. (c) High-resolution XPS for the $\mathrm{Cl}$ $2 p$ region of the chlorinated graphene produced with the $\mathrm{NaCl}$ solution in (b).
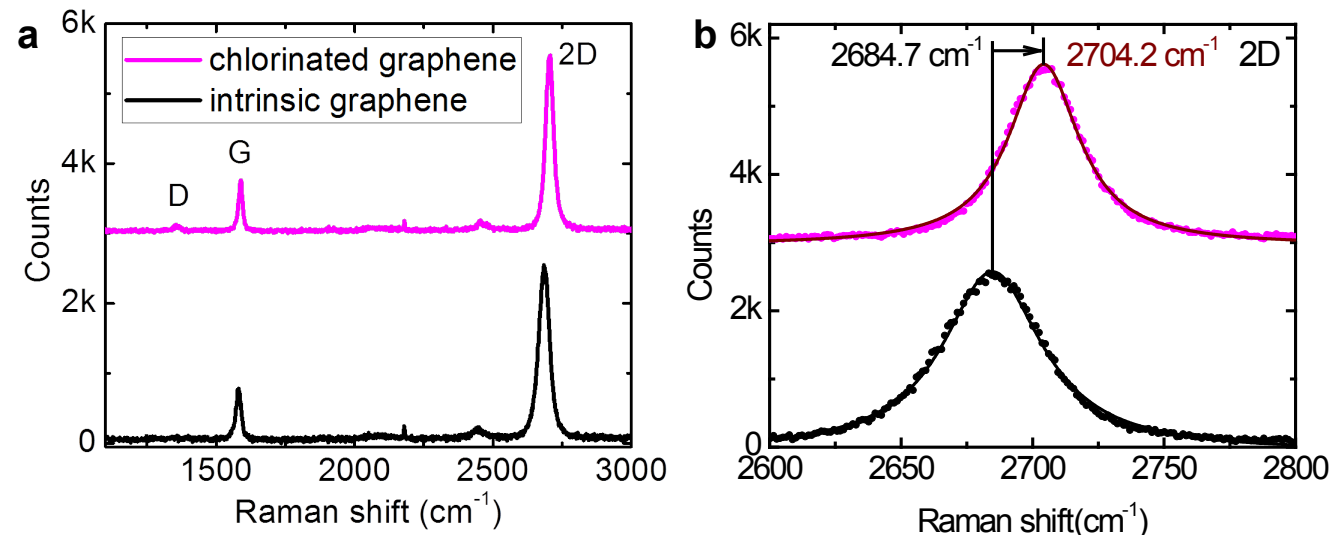

Figure S2. Raman spectra of a monolayer graphene sample on a $\mathrm{SiO}_{2} / \mathrm{Si}$ substrate before (black) and after (magenta) chlorination at $1.6 \mathrm{~V}$ (vs. Ag/AgCl) for $4 \mathrm{~min}$. (a) Full spectra. (b) Zoom-in of the 2D band, showing a substantial blue shift after chlorination. 


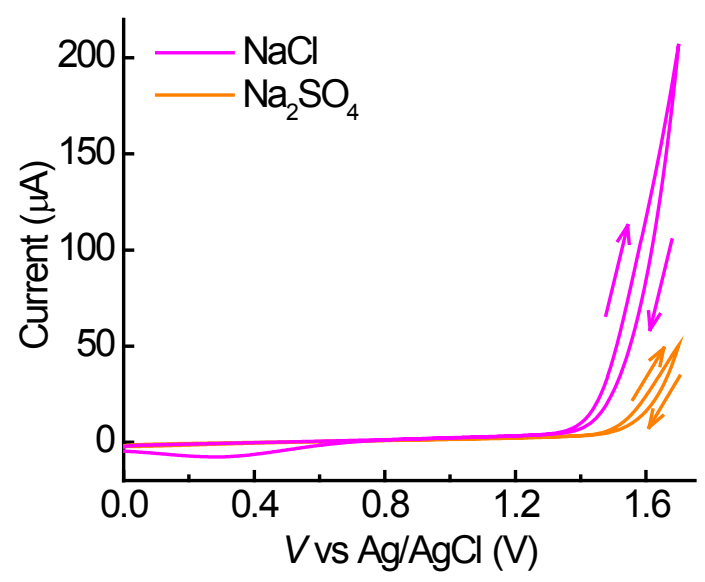

Figure S3. Cyclic voltammograms of a graphene electrode in a $\mathrm{pH}=3$ buffer with the addition of $\mathrm{NaCl}$ or $\mathrm{Na}_{2} \mathrm{SO}_{4}$ to the same ionic strength. A three-electrode configuration was used with graphene as the working electrode, a Pt wire as the counter electrode, and an $\mathrm{Ag} / \mathrm{AgCl}$ electrode as the reference electrode. The same monolayer graphene electrode was measured in $200 \mathrm{mM} \mathrm{pH}=3$ phosphate buffers with the addition of $1.5 \mathrm{M} \mathrm{NaCl}$ (magenta curve) vs. with the addition of $0.5 \mathrm{M} \mathrm{Na}_{2} \mathrm{SO}_{4}$ (orange curve). Scanning rate: $19 \mathrm{mV} / \mathrm{s}$. 

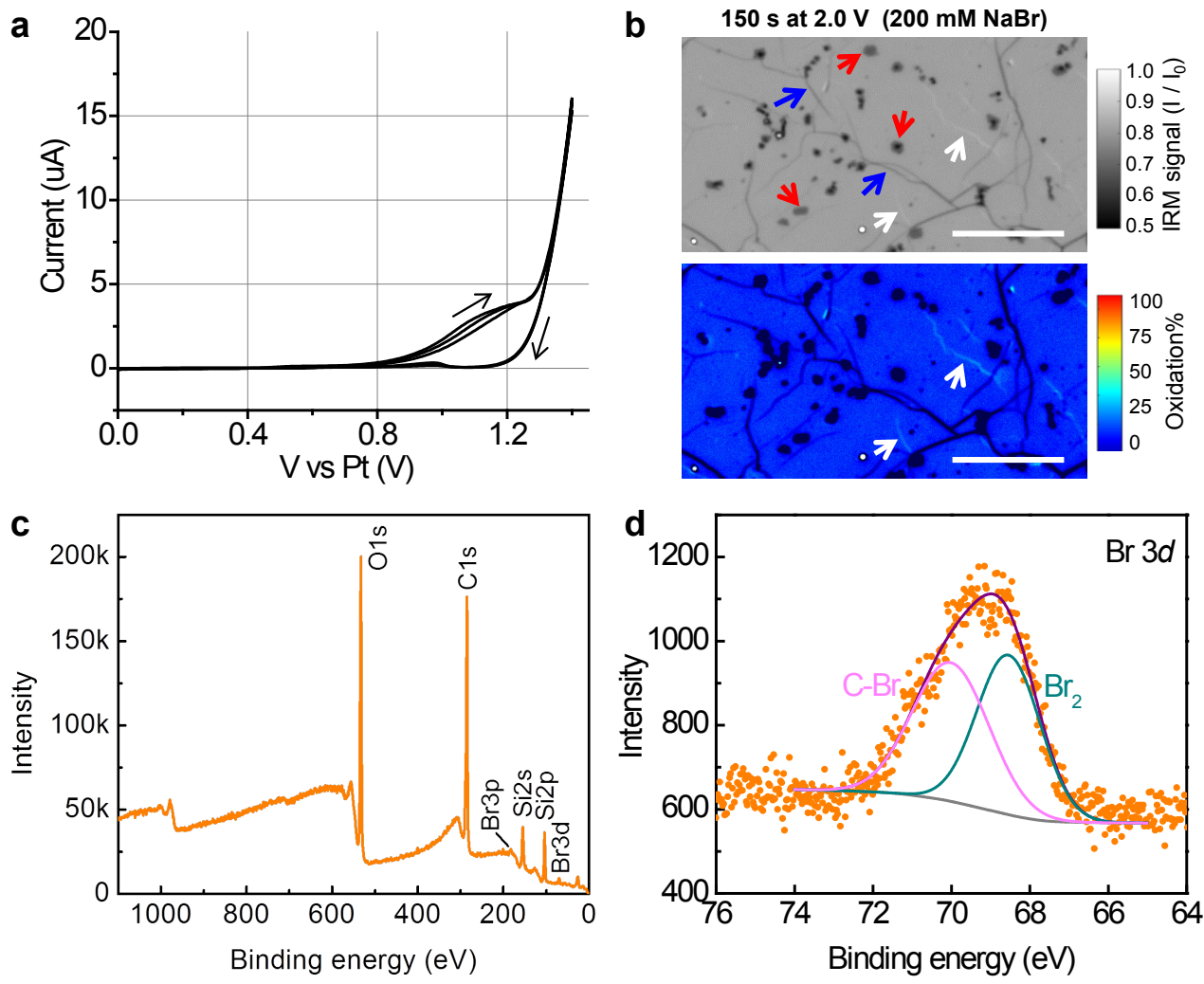

Figure S4. Electrochemical bromination of graphene in an aqueous $\mathrm{NaBr}$ solution. (a) Cyclic voltammograms measured with a graphene electrode in a $\mathrm{pH}=3$ phosphate buffer containing $1 \mathrm{M} \mathrm{NaBr}$. Electrochemical current due to the oxidation of $\mathrm{Br}^{-}$rose at $\sim 0.8 \mathrm{~V}$ (vs. Pt). (b) In situ IRM images (top) and the converted map of local oxidation degree (bottom) for a graphene sample on a glass substrate after electrochemical reaction at $2.0 \mathrm{~V}$ vs. Pt for $150 \mathrm{~s}$ in a pH = 3 phosphate buffer containing $200 \mathrm{mM} \mathrm{NaBr}$. Electrochemical oxidation of graphene was completed suppressed. Arrows: wrinkles (blue), sporadic bilayer islands (red), and minor cracks in the monolayer graphene (white). The IRM signal does not faithfully reflect the local oxidation degree at these structural defects. Scale bars: $10 \mu \mathrm{m}$. (c) Survey-mode XPS spectra of monolayer graphene on a $\mathrm{Si} / \mathrm{SiO}_{2}$ substrate after electrochemical reaction at $1.6 \mathrm{~V}$ vs $\mathrm{Pt}$ for $4 \mathrm{~min}$ in a $\mathrm{pH}=3$ phosphate buffer containing $1 \mathrm{M} \mathrm{NaBr}$. (d) A detailed scan of the $\mathrm{Br} 3 d$ signal. This signal is fitted by two peaks centered at $70.2 \mathrm{eV}$ and $68.5 \mathrm{eV}$, attributable to the $\mathrm{C}$ $\mathrm{Br}$ bond in brominated graphene and physically adsorbed $\mathrm{Br}_{2}$, respectively. ${ }^{6-8}$ Quantification of the signal areas indicated a total $\mathrm{Br}: \mathrm{C}$ atomic ratio of $0.8 \%$, with bonded and adsorbed $\mathrm{Br}$ each at $\sim 0.4 \%$. 

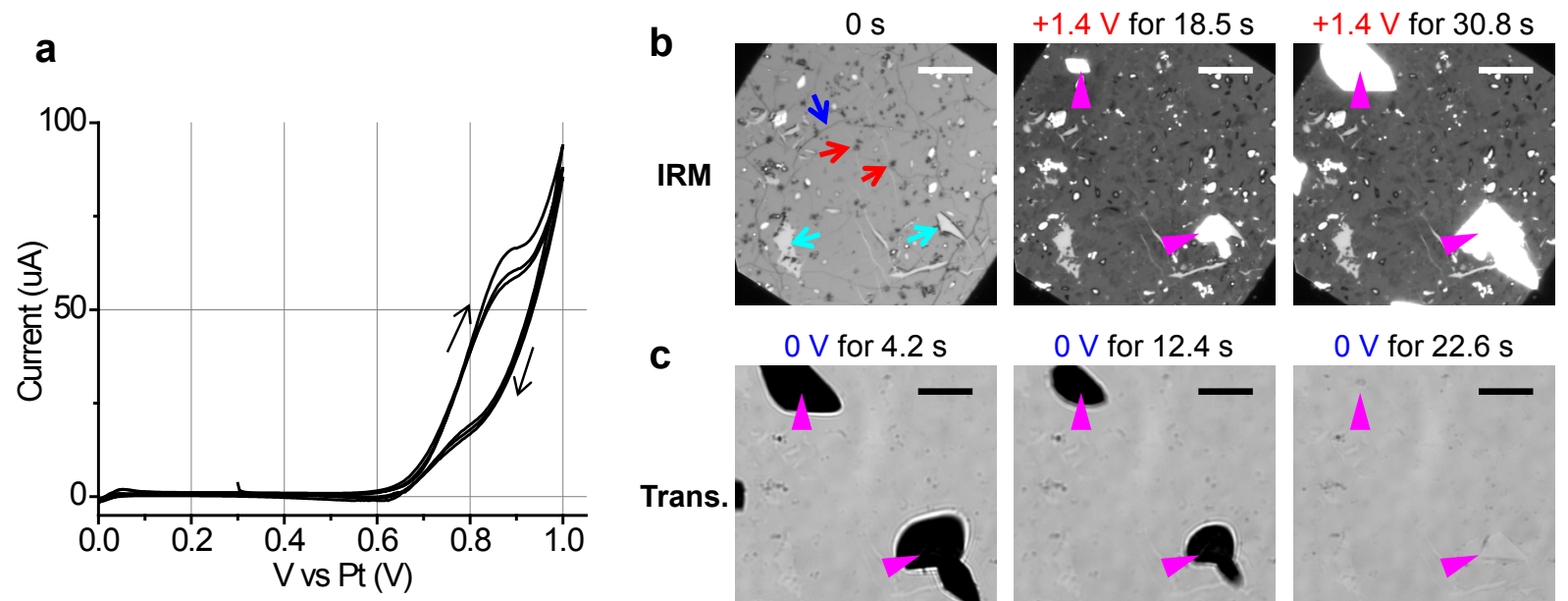

C

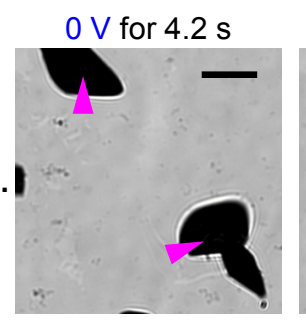

$0 \mathrm{~V}$ for $12.4 \mathrm{~s}$

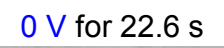

Trans.
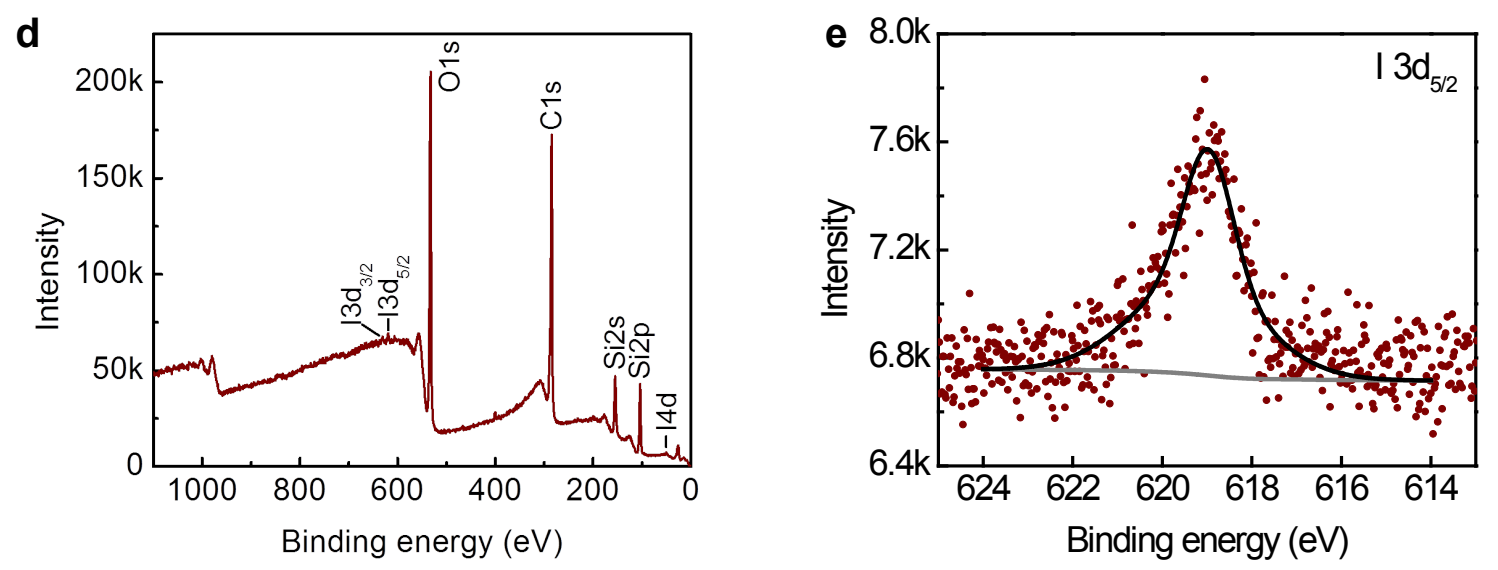

Figure S5. Electrochemical iodination of graphene in an aqueous KI solution. (a) Cyclic voltammograms measured with a graphene electrode in a $\mathrm{pH}=3$ phosphate buffer containing $200 \mathrm{mM} \mathrm{KI}$. Electrochemical current due to the oxidation of $\mathrm{I}^{-}$rose at $\sim 0.6 \mathrm{~V}$. (b) In situ IRM image for a graphene sample on a glass substrate, before (left) and after electrochemical reaction at $1.4 \mathrm{~V}$ (vs. Pt) in a pH = 3 phosphate buffer containing $200 \mathrm{mM} \mathrm{KI}$ for $18.5 \mathrm{~s}$ (center) and $30.8 \mathrm{~s}$ (right). Arrows: wrinkles (blue), sporadic bilayer islands (red), and minor tears (cyan) in the monolayer graphene. Magenta arrowheads: growth of $\mathrm{I}_{2}$ islands on the graphene surface. The IRM images overall darkened due to the generation of dissolved $\mathrm{I}_{2}$ and $\mathrm{I}_{3}{ }^{-}$in the solution, yet became exceedingly bight locally for the deposited $I_{2}$ islands owing to reflection. Scale bars: $10 \mu \mathrm{m}$. (c) Same sample, next under transmission microscopy and with the application of a reductive voltage of $0 \mathrm{~V}$ (vs. Pt) for $12.4 \mathrm{~s}$ and $22.6 \mathrm{~s}$. Magenta arrowheads point to the deposited $\mathrm{I}_{2}$ islands on the graphene surface, which appeared dark due to the absorption of light, and were quickly reduced under the reductive voltage. Scale bars: $10 \mu \mathrm{m}$. (d) Survey-mode XPS spectra of monolayer graphene on a $\mathrm{Si} / \mathrm{SiO}_{2}$ substrate after electrochemical reaction in a $\mathrm{pH}=3$ phosphate buffer containing $1 \mathrm{M} \mathrm{KI}$ at $1.4 \mathrm{~V}$ vs. Pt for 4 min. (e) Detailed scan of the I $3 d_{5 / 2}$ signal. An I:C atomic ratio of $0.1 \%$ was calculated from the areas of $13 d_{5 / 2}$ and $C 1 s$ peaks. 


\section{Reference for Supporting Information}

(1) Liang, X.; Sperling, B. A.; Calizo, I.; Cheng, G.; Hacker, C. A.; Zhang, Q.; Obeng, Y.; Yan, K.; Peng, H.; Li, Q.; Zhu, X.; Yuan, H.; Hight Walker, A. R.; Liu, Z.; Peng, L.-m.; Richter, C. A. Toward clean and crackless transfer of graphene. ACS Nano 2011, 5, 9144-9153.

(2) Hofmann, S. Auger- and X-Ray Photoelectron Spectroscopy in Materials Science; Springer Berlin: Heidelberg, 2013.

(3) Li, W.; Moon, S.; Wojcik, M.; Xu, K. Direct optical visualization of graphene and its nanoscale defects on transparent substrates. Nano Lett. 2016, 16, 5027-5031.

(4) Wojcik, M.; Li, Y.; Li, W.; Xu, K. Spatially resolved in situ reaction dynamics of graphene via optical microscopy. J. Am. Chem. Soc. 2017, 139, 5836-5841.

(5) Li, W.; Wojcik, M.; Xu, K. Optical microscopy unveils rapid, reversible electrochemical oxidation and reduction of graphene. Nano Lett. 2019, 19, 983-989.

(6) Zheng, J.; Liu, H. T.; Wu, B.; Di, C. A.; Guo, Y. L.; Wu, T.; Yu, G.; Liu, Y. Q.; Zhu, D. B. Production of graphite chloride and bromide using microwave sparks. Sci. Rep. 2012, 2, 662.

(7) Jankovský, O.; Šimek, P.; Klimová, K.; Sedmidubský, D.; Matějková, S.; Pumera, M.; Sofer, Z. Towards graphene bromide: bromination of graphite oxide. Nanoscale 2014, 6, 6065-6074.

(8) Au, H.; Rubio, N.; Shaffer, M. S. P. Brominated graphene as a versatile precursor for multifunctional grafting. Chem. Sci. 2018, 9, 209-217. 